\title{
Evaluating Value-at-Risk Models with Desk-Level Data ${ }^{\#}$
}

\author{
Jeremy Berkowitz - University of Houston* \\ jberkowitz@uh.edu \\ Peter Christoffersen - McGill University \\ peter.christoffersen@mcgill.ca \\ Denis Pelletier - North Carolina State University \\ denis_pelletier@ncsu.edu
}

Preliminary Version: October 6, 2005

\begin{abstract}
We present new evidence on disaggregated profit and loss and VaR forecasts obtained from a large international commercial bank. Our dataset includes daily $\mathrm{P} / \mathrm{L}$ generated by four separate business lines within the bank. All four business lines are involved in securities trading and each is observed daily for a period of at least two years. We also collected the corresponding daily, 1-day ahead VaR forecasts for each business line. Given this rich dataset, we provide an integrated, unifying framework for assessing the accuracy of $\mathrm{VaR}$ forecasts. Our approach includes many existing backtesting techniques as special cases. In addition, we describe some new tests which are suggested by our framework. A thorough Monte Carlo comparison of the various methods is conducted to provide guidance as to which of these many tests have the best finite-sample size and power properties.
\end{abstract}

\# Christoffersen acknowledges financial support from FQRSC, IFM2, and SSRHC and Pelletier acknowledges financial support from the North Carolina State University Edwin Gill Research Grant.

* Address correspondence to: Jeremy Berkowitz, Bauer College of Business, 334 Melcher Hall, University of Houston, Houston, Texas, 77204. 


\section{Introduction}

Value-at-Risk (VaR) is by far the leading measure of portfolio risk in use at major commercial banks. Despite criticisms of VaR's statistical properties (e.g. Artzner, Delbaen, Eber, and Heath (1999)) and theoretical concerns that widespread usage of VaR could increase systemic risk (Basak and Shapiro (2002)), financial institutions and their regulators increasingly rely on $\mathrm{VaR}$ as a measure of portfolio risk.

Under the "internal models approach" of the Basle Accord on banking (Basle Committee on Banking Supervision, 1996), financial institutions have the freedom to specify their own model to compute their Value-at-Risk. The accuracy of VaR models can be checked and must be checked by assessing the accuracy of the forecasts - a procedure known as backtesting.

Model validation in general and backtesting in particular is an important component of the Supervisory Review Process (the Second Pillar) in Basel II (Basle Committee on Banking Supervision, 2004). The lively public policy debate sparked by Basle II has focused attention on banks' procedures for backtesting. While no particular technique for backtesting is currently suggested in Basle II, the potential for a supervisor endorsed backtesting technique has clear implications for the banking system as a whole. It is thus crucially important for the institutions and regulators to assess the quality of the models employed.

In this paper, we provide further empirical evidence on the accuracy of actual VaR models in forecasting portfolio risk. We obtained the daily profit and loss $(\mathrm{P} / \mathrm{L})$ generated by four separate business lines from a large, international commercial bank. Each of the business line's P/L series is observed daily for a period of more than two years. For two of the business lines, we have over 600 daily observations while for the other two we have over 800 observations yielding a panel of 2,930 observations.

All four business lines are involved in securities trading but the exact nature of each business line is not known to us. Each series is constructed and defined in a consistent manner but the series are normalized to protect the bank's anonymity. We do not observe the aggregate P\&L summed across the business desks.

In addition to the daily $\mathrm{P} / \mathrm{L}$ data, we obtained the corresponding daily, 1-day ahead VaR forecasts. For each business line within the bank, and for each day, the VaR 
forecasts are estimates of the $1 \%$ lower tail. Our data set complements that of Berkowitz, O'Brien (2002) who obtained daily bank-wide P/L and VaR data, but who were not able to obtain any information on separate business lines within the same bank.

Given this rich dataset, we provide an integrated, unifying framework for assessing the accuracy of VaR forecasts. Our approach includes the existing tests proposed Christoffersen (1998) and Christoffersen and Pelletier (2004) as special cases. In addition, we describe some new tests which are suggested by our framework.

In order to provide some guidance as to which of these many tests have the best finite-sample size and power properties, we conduct a thorough horserace in a Monte Carlo setting.

Testing the accuracy of a Value-at-Risk (VaR) model is based on the observation that the VaR forecast is a (one-sided) interval forecast. Violations - the days on which portfolio losses exceed the VaR - should therefore be unpredictable. In particular, the violations form a martingale difference sequence. The martingale hypothesis has a long and distinguished history in economics and finance (Durlauf (1991)). Related work dates back at least to the random walk theory of stock prices. The risk-neutral pricing methods of Harrison and Kreps (1979) and Harrison and Pliska (1981) are based on the martingale representation theorem.

As a result of this extensive toolkit, we are able to cast all existing methods of evaluating VaR under a common umbrella of martingale tests. This immediately suggests several testing strategies. The most obvious is a test of whether any of the autocovariances are nonzero. The standard approach to test for uncorrelatedness is by estimating the sample autocovariances or sample autocorrelations. In particular, we suggest the well-known Ljung-Box test of the violation sequence's autocorrelation function.

The second set of tests are inspired by Campbell and Shiller (1987) and Engle and Manganelli (2004). If the violations are a martingale difference sequence, then they should be uncorrelated by any transformation of the variables available when the VaR is computed. It suggests a regression of the violations/non-violations on their lagged values and lagged variables such as previous VaRs. 
A third set of tests are adapted from Christoffersen and Pelletier (2004) who focus on hazard rates and durations. These tests are based on the observation that the number of days separating the violations (i.e., the durations) should be totally unpredictable.

Lastly, a fourth set of tests is taken from Durlauf (1991). He derives a set of tests of the martingale hypothesis based on the spectral density functions. This approach has several features to commend it. Unlike variance ratio tests, spectral tests have power against any linear alternative of any order. Spectral density tests have power to detect any second moment dynamics. Variance ratio tests are typically not consistent against all such alternatives.

Because the violation of the $\mathrm{VaR}$ is, by construction, a rare event, the effective sample size in realistic risk management settings can be quite small. It follows that we can't rely on the asymptotic distribution of the tests to conduct inference. We instead rely on Dufour (2004)'s Monte Carlo testing technique which yields tests with exact level, irrespective of the sample size and the number of replications used.

The paper proceeds as follows. In Section 2 we present some new evidence on desk-level P/Ls and VaRs from a large international bank and we discuss the pros and cons of market risk management using historical simulation. Section 3 gives an overview of existing methods for backtesting VaR estimates and it suggests a few new approaches as well. Section 4 presents the results of a detailed horserace among the methods in terms of size and power properties in finite sample. Section 5 presents the empirical results of applying the various testing methods to our desk-level data sample. Section 6 concludes.

\section{Desk Level P\&L and VaR at a Commercial Bank}

We collected the daily profit and loss (P/L) generated by four separate business lines from a large, international commercial bank. All four business lines are involved in securities trading but the exact nature of each business line is not known to us. Each series is constructed and defined in a consistent manner but they series are normalized to protect the bank's anonymity. We do not observe the aggregate P\&L summed across the business desks. 
In addition to the daily revenue data, we obtained the corresponding 1-day ahead Value-at-Risk forecasts. The VaR forecasts are estimates of the $99 \%$ lower tail and are calculated for each business line within the bank.

Suppose revenue is denoted by $R_{t}$. The $p$ percent Value-at-Risk (VaR) is the quantity $V_{a} R_{t}$ such that

$$
F\left(R_{t+1}^{<-V a R_{t}} \mid \Omega_{t}\right)=p
$$

where $\Omega_{t}$ is the time-t information set. The $\mathrm{VaR}$ is the $p^{\text {th }}$ percentile of the return distribution. The probability $p$ is referred to as the coverage rate. By definition, the coverage rate is the probability that the lower tail VaR will be exceeded on a given day.

In our dataset the tail percentile of the bank's VaR is set at $p=.01$ which yields a one-sided, 99\% confidence interval. This is quite far in the tail but is typical of the VaR forecasts at commercial bank (e.g., Berkowitz and O'Brien (2002)).

The daily P/L (dashed) and associated (negative) VaR (solid) are plotted over time in Figure 1. Business line 1 is observed from January 2, 2001 through June 30, 2004, business line 2 is observed from April 2, 2001 and lines 3 and 4 from January 3, 2002. Several interesting observations are apparent in Figure 1. First, notice that bursts of volatility are apparent in each of the P/L series (e.g. mid-sample for line 1 and endsample for line 2) but these bursts are not necessarily synchronized across business lines. Second, note the occasional and very large spikes in the P/Ls. These are particularly evident for line 1 and 2. Third, the bank VaRs exhibit considerable short-term variability (line 3), sometimes they show persistent trends away from the P/Ls (line 1) and even what looks like regime-shifting without corresponding moves in the associated P/L (line 2).

Table 1 reports the first four sample moments of the P/Ls and VaRs along with the exact number of daily observations. Of particular interest are the skewness and kurtosis estimates. Skewness is evident in business line 1 (negative) and line 2 (positive) but much less so in business lines 3 and 4. Excess kurtosis is evident in all four business lines and dramatically so in lines 1 and 2. The skewness statistics confirm the occasional spikes in the P/Ls in Figure 1. For completeness, the descriptive statistics for the VaRs are also reported in Table 1. 
The occasional bursts of volatility apparent in the P/Ls in Figure 1 are explored further in Figure 2 where we demean the P/Ls and plot their daily absolute values over time. While the spikes in $\mathrm{P} / \mathrm{Ls}$ dominate the pictures, episodes of high volatility is evident in each of the series, although perhaps less so in business line 3 .

\section{A Unified Framework for VaR Evaluation}

Under the Market Risk Amendment to the Basle Accord effective in 1996, qualifying financial institutions have the freedom to specify their own model to compute their Value-at-Risk. It thus becomes crucially important for regulators to assess the quality of the models employed by assessing the forecast accuracy—a procedure known as "backtesting".

The accuracy of a set of VaR forecasts can be assessed by viewing them as onesided interval forecasts. A violation of the $\mathrm{VaR}$ is defined as occurring when the ex post return is lower than the VaR. Specifically, we define violations

$$
I_{t+1}=\left\{\begin{array}{l}
1, \quad \text { if } R_{t+1}<-\operatorname{VaR}_{t}(p) \\
0, \quad \text { else }
\end{array}\right.
$$

i.e. a sequence of zeros and ones. By definition, the conditional probability of violating the VaR should always be

$$
\operatorname{pr}\left(I_{t+1}=1 \mid \Omega_{t}\right)=p
$$

for every time-t. Below, we will refer to tests of this property as conditional coverage (CC) tests.

\section{A. Autocorrelation Tests}

Christoffersen (1998) notes that property (3) implies that any sequence of violations, $\left\{I_{t}\right\}$, should be an i.i.d. Binomial random variable with mean $p$. In order to formally test this, Christoffersen (1998) embeds the null hypothesis of an i.i.d. Binomial within a general first-order Markov process.

If $\left\{I_{t}\right\}$ is a first-order Markov process the one-step-ahead transition probabilities $\operatorname{pr}\left(I_{t+1} \mid I_{t}\right)$ are given by 


$$
\left[\begin{array}{ll}
1-\pi_{01} & \pi_{01} \\
1-\pi_{11} & \pi_{11}
\end{array}\right]
$$

where $\pi_{i j}$ is the transition $\operatorname{pr}\left(I_{t+1}=j \mid I_{t}=i\right)$.

Under the null, the violations have a constant conditional mean which implies the two linear restrictions, $\pi_{01}=\pi_{11}=p$. A likelihood ratio test of these restrictions can be computed from the likelihood function

$$
L\left(I ; \pi_{01}, \pi_{11}\right)=\left(1-\pi_{01}\right)^{T_{0}-T_{01}} \pi_{01}^{T_{01}}\left(1-\pi_{11}\right)^{T_{1}-T_{11}} \pi_{11}^{T_{11}}
$$

where $T_{i j}$ denotes the number of observations with a $j$ following a $i$ and $T_{i}$ is the number of $i$ is the number of ones or zeros in the sample.

We note that all the tests are carried out conditioning on the first observation. The tests are asymptotically distributed as chi-square with degree of freedom two. But as for all the tests we will rely on finite sample p-values as discussed below.

In this paper, we extend and unify the existing tests by noting that the de-meaned violations $\left\{I_{t}-p\right\}$ form a martingale difference sequence. By definition of the violation, equations (2)-(3) immediately imply that

$$
E\left[\left(I_{t+1}-p\right) \mid \Omega_{t}\right]=0
$$

where $\Omega_{t}$ is the information set up to time- $t$. The de-meaned violations form a martingale difference sequence (m.d.s.) with respect to the agent's time-t information set. This will be an extremely useful property because it implies that the violation sequence is uncorrelated at all leads and lags.

This motivates a variety of tests which focus on the white noise or martingale property of the sequence. Since white noise has zero autocorrelations at all leads and lags, the violations can be tested by calculating statistics based on the sample autocorrelations.

For any variable $Z_{t}$ in the agent's time-t information set,

$$
E\left[\left(I_{t+1}-p\right) \otimes Z_{t}\right]=0
$$

which is familiar as the basis of GMM estimation.

Specifying $Z_{t}$ to be the most recent de-meaned violation, we have

$$
E\left[\left(I_{t+1}-p\right)\left(I_{t}-p\right)\right]=0 .
$$


The violation sequence has a first-order autocorrelation of zero, under the null. It is this property which is exploited by the Markov test of Christoffersen (1998).

More generally, if we set $Z_{t}=I_{t-k}$ for any $k \geq 0$,

$$
E\left[\left(I_{t+1}-p\right)\left(I_{t-k}-p\right)\right]=0
$$

which says that the de-meaned violation sequence is in fact White Noise. We write this null hypothesis compactly as

$$
\left(I_{t+1}-p\right) \stackrel{\text { iid }}{\sim}(0, p(1-p)) .
$$

A natural testing strategy is to check whether any of the autocorrelations are not zero. Under the null all the autocorrelations are zero

$$
H_{0}: \gamma_{k}=0, \quad k>0
$$

and the alternative hypothesis of interest is that

$$
H_{a}: \gamma_{k} \neq 0 \text {, for some } k \text {. }
$$

The Portmanteau or Ljung-Box statistics, for example, have known distribution which can be compared to critical values under the white noise null. The Ljung-Box statistic is a joint test of whether the first $m$ autocorrelations are zero. We can immediately make this into a test of of a VaR model by calculating the autocorrelations of $\left(I_{t+1}-p\right)$ and then calculating

$$
L B(m)=T(T+2) \sum_{k=1}^{m} \frac{\gamma_{k}^{2}}{T-k}
$$

which is asymptotically chi-square with $m$ degrees of freedom.

We may also want to consider whether violations can be predicted by including other data in the information set such as past returns. Under the null hypothesis, it must be that

$$
E\left[\left(I_{t+1}-p\right) g\left(I_{t}, I_{t-1}, \ldots, R_{t}, R_{t-1}, \ldots\right)\right]=0 .
$$

for any non-anticipating function $g(\cdot)$.

In analogy with Engle and Manganelli (2004), we might consider the $n$ th-order autoregression

$$
I_{t}=\alpha+\sum_{k=1}^{n} \beta_{1 k} I_{t-k}+\sum_{k=1}^{n} \beta_{2 k} g\left(I_{t-k}, I_{t-k-1}, \ldots, R_{t-k}, R_{t-k-1}, \ldots\right)+u_{t}
$$


where we set $g\left(I_{t-k}, I_{t-k-1}, \ldots, R_{t-k}, R_{t-k-1}, \ldots\right)=V a R_{t-k+1}$ and $n=1$. We can then test if the coefficients $\beta$ are statistically significant and whether $\alpha=p$ using standard $t$-tests. We refer to this test as the CaViaR test of Engle and Manganelli.

\section{B. Hazard Rates and Tests for Clustering in Violations}

Under the null that VaR forecasts are correctly specified, the violations should occur at random time intervals. Suppose the duration between two violations is defined as

$$
D_{i}=t_{i}-t_{i-1}
$$

where $t_{i}$ denotes the day of the violation number $i$. The duration between violations of the VaR should be completely unpredictable. There is an extensive literature on testing duration dependence (e.g., Kiefer (1988), Engle and Russel (1998), Gourieroux (2000)) which makes this approach particularly attractive.

Christoffersen and Pelletier (2004) apply duration-based tests to the problem of assessing VaR forecast accuracy. In this section we expand upon their methods. The duration-based tests can be viewed as another procedure for testing whether the violations form a martingale difference sequence.

Using the Binomial property, the probability of a violation next period is exactly equal to $\operatorname{pr}\left(D_{i}=1\right)=\operatorname{pr}\left(I_{t+1}=1\right)=p$. The probability of a violation in $d$ periods is

$$
\operatorname{pr}\left(D_{i}=d\right)=\operatorname{pr}\left(I_{t+1}=0, I_{t+2}=0, \ldots, I_{t+d}=1\right) .
$$

Under the null of an accurate $\mathrm{VaR}$ forecast, the violations are distributed

$$
I_{t+1} \sim \text { iid }(p, p(1-p)) \text {. }
$$

This allows us to rewrite (13) as

$$
\begin{aligned}
\operatorname{pr}\left(D_{i}=d\right) & =(1-p) \ldots(1-p)(p) \\
& =(1-p)^{d-1} p .
\end{aligned}
$$

Equation (14) says that the density of the durations declines geometrically under the null hypothesis.

A more convenient representation of the same information is given by transforming the geometric probabilities into a flat function. The hazard rate defined as 


$$
\lambda\left(D_{i}\right)=\frac{\operatorname{pr}\left(D_{i}=d\right)}{1-\operatorname{pr}\left(D_{i}<d\right)}
$$

is such a transformation. Writing out the hazard function $\lambda\left(D_{i}\right)$ explicitly

$$
\frac{(1-p)^{d-1} p}{1-\sum_{j=0}^{d-2}(1-p)^{j} p}=p
$$

collapses to a constant after expanding and collecting terms.

We conclude that under the null, the hazard function of the durations should be flat and equal to $p$. Tests of this null are constructed by Christoffersen and Pelletier (2004). They consider alternative hypothesis under which the violation sequence, and hence the durations, display dependence or clustering. The only (continuous) random distribution is the exponential, thus under the null hypothesis the distribution of the durations should be

$$
f_{\text {exp }}(D ; p)=p e^{-p D}
$$

The most powerful of the two alternative hypotheses they consider is that the durations follow a Weibull distribution where

$$
f_{W}(D ; a, b)=a^{b} b D^{b-1} \exp ^{-(a D)^{b}}
$$

This distribution is able to capture violation clustering. When $b<1$, the hazard, i.e. the probability of getting a violation at time $D_{i}$ given that we did not up to this point, is a decreasing function of $D_{i}$.

It is also possible to capture duration dependence without resorting to the use of a continuous distribution. We can introduce duration dependence by having non-constant probabilities of a violation,

$$
\begin{aligned}
\operatorname{pr}\left(D_{i}=d\right) & =\operatorname{pr}\left(I_{t+1}=0, I_{t+2}=0, \ldots, I_{t+d}=1\right) \\
& =\left(1-p_{1}\right)\left(1-p_{2}\right) \cdots\left(1-p_{d-1}\right) p_{d}
\end{aligned}
$$

where

$$
p_{d}=\operatorname{pr}\left(I_{t+d}=1 \mid I_{t+d-1}=0, \ldots, I_{t+1}=0\right)
$$

In this case, one must specify how these probabilities $p_{d}$ vary with $d$. We will set

$$
p_{d}=a d^{b}
$$


with $b \leq 0$ in order to implement the test. We refer to this as the Geometric test below.

Except for the first and last duration the procedure is straightforward, we just count the number of days between each violation. We then define a binary variable $C_{i}$ which tracks whether observation $i$ is censored or not. Except for the first and last observation, we always have $C_{i}=0$. For the first observation if the hit sequence starts with 0 then $D_{1}$ is the number of days until we get the first hit. Accordingly $C_{1}=1$ because the observed duration is left-censored. The procedure is similar for the last duration. If the last observation of the hit sequence is 0 then the last duration, $D_{N(T)}$, is the number of days after the last 1 in the hit sequence and $C_{N(T)}=1$ because the spell is right-censored.

The contribution to the likelihood of an uncensored observation is its corresponding p.d.f. For a censored observation, we merely know that the process lasted at least $D_{1}$ or $D_{N(T)}$ days so the contribution to the likelihood is not the p.d.f. but its survival function $S\left(D_{i}\right)=1-F\left(D_{i}\right)$. Combining the censored and uncensored observations, the log-likelihood is

$$
\begin{aligned}
\ln L(D ; a, b)= & C_{1} \ln S\left(D_{1}\right)+\left(1-C_{1}\right) \ln f\left(D_{1}\right)+\sum_{i=2}^{N(T)-1} \ln f\left(D_{i}\right) \\
& +C_{N(T)} \ln S\left(D_{N(T)}\right)+\left(1-C_{N(T)}\right) \ln f\left(D_{N(T)}\right)
\end{aligned}
$$

Once the durations are computed and the truncations taken care of, then the likelihood ratio tests can be calculated in a straightforward fashion. The null and alternative hypotheses for the test is

$$
\begin{aligned}
& H_{0}: \quad b=1 \& a=p \\
& H_{a}: \quad b \neq 1 \& a \neq p
\end{aligned}
$$

The only added complication is that the ML estimates are no longer available in closed form, they must be found using numerical optimization.

\section{Spectral Density Tests}

A final method for testing the martingale property is to examine the shape of the spectral density function. There is a long standing literature on using the spectral density 
for this purpose because white noise has a particularly simple representation in the frequency domain -- its spectrum is a flat line (e.g., Durlauf (1991)). Statistical tests are constructed by examining if the sample spectrum is "close" to the theoretical flat line.

The spectral density function is defined as a transformation of the autocovariance sequence,

$$
f(\omega)=\frac{1}{2 \pi} \sum_{k=-\infty}^{\infty} \gamma_{k} e^{-i k \omega} .
$$

For a white noise process, all the autocovariances equal zero for any $k \neq 0$. This means that for the hit sequence the spectral density collapses to

$$
f(\omega)=\frac{1}{2 \pi} p(1-p)
$$

for all $\omega \in[0, \pi]$.

The spectral density function is constant and proportional to the variance.

Equivalently, the spectral distribution function is a $45^{\circ}$ line. The asymptotic theory centers on the convergence of the random, estimated spectral density function using a functional central limit theorem.

The sample spectrum (or periodogram) is given by replacing the population autocovariances with the finite-sample estimates,

$$
\hat{f}(\omega)=\frac{1}{2 \pi} \sum_{k=-\infty}^{\infty} \hat{\gamma}_{k} e^{-i k \omega}
$$

which should be approximately a flat line.

It is often convenient to de-mean the sample spectral density and take the partial sums

$$
\hat{U}(\omega)=\sum_{\omega=0}^{\pi}\left(\frac{\hat{f}(\omega)}{\hat{\sigma}^{2}}-\frac{1}{\pi}\right)
$$

for each frequency $\omega \in[0, \pi]$. The $\hat{U}(\omega)$ are deviations of the sample spectral distribution from the 45 degree line. If the violations are white noise, the deviations should be small.

Durlauf (1991) derives the asymptotic distribution of a variety of statistics based on these deviations. The Cramér-Von Mises (CVM) test statistic is the sum of squared deviations 


$$
C V M=\sum_{\omega=0}^{\pi} \hat{U}(\omega)^{2}
$$

and it converges to a known distribution whose critical values can be tabulated numerically.

Another common test statistic dates to Bartlett, who showed the supremum

$$
\sup _{\omega} \hat{U}(\omega)^{2}
$$

converges to the Kolmogorov-Smirnov (KS) statistic.

These test statistics have several attractive features. Unlike some tests of white noise (e.g., variance ratio tests), spectral tests have power against any linear alternative of any order. That is, the test has power to detect any second moment dynamics (see Durlauf, (1991)). Both the CVM and KS statistics diverge asymptotically if $I_{t}$ is any stationary process which is not white noise.

\section{Size and Power Properties}

Given the large variety of backtesting procedures surveyed in section 3, it is important to give users guidance as to their comparative size and power properties in a controlled setting.

\section{A. Effective Size of the Tests}

In order to assess the size properties of the various methods, we simulate i.i.d. Bernoulli samples with probabilities $\mathrm{p}=1 \%$ and $5 \%$ respectively. For each Bernoulli probability, we consider several different sample sizes, from 250 to 1500 . Rejection rates under the null are calculated over 10,000 Monte Carlo trials. If the asymptotic distribution is accurate in the sample sizes considered then the rejection frequencies should be close to the nominal size of the test, which we set to $10 \%$.

Table 2 contains the actual size of the conditional coverage (CC) tests when the asymptotic critical values are used. The number of observations in each simulated sample is reported in the first column. The top panel shows the finite sample test sizes for a $1 \%$ VaR. We see that the $\mathrm{LB}(1)$ test tends to be undersized and the $\mathrm{LB}(5)$ tends to be oversized. The Markov test tends to be undersized and the Weibull test tends to be oversized. The Geometric test is extremely oversized for the smallest sample. The Caviar 
and CVM tests are undersized for the smallest sample size and somewhat oversized for larger samples. Finally, the KS test has good size beyond the smallest sample sizes where it is undersized.

The results in the bottom panel cover the 5\% VaR. In this case the $\mathrm{LB}(1)$ test is slightly undersized whereas the $\operatorname{LB}(5)$ is very close to the desired $10 \%$. The Markov and Weibull tests are both oversized. The Geometric is somewhat undersized, whereas the Caviar, KS and CVM tests now are very close to the desired 10\% level.

The overall conclusion from Table 2 is that for small sample sizes and for the $1 \%$ VaR which is arguably the most common in practice, the asymptotic critical values can be highly misleading. When computing power below we therefore rely on the Dufour (2004) Monte Carlo testing technique which is described in detail in Section 5.

\section{B. Finite Sample Power of the Tests}

In order to perform a power comparison, we use a flexible and simple GARCH specification as a model of the $\mathrm{P} / \mathrm{L}$ process. We estimate the parameters for each business line separately in order to model the volatility persistence in each series.

The GARCH model allows for an asymmetric volatility response or "leverage effect". In particular, we use the NGARCH(1,1)-t(d) specification,

$$
\begin{aligned}
& \mathrm{R}_{\mathrm{t}+1}=\sigma_{\mathrm{t}+1}((\mathrm{~d}-2) / \mathrm{d})^{1 / 2} \mathrm{z}_{\mathrm{t}+1} \\
& \sigma_{\mathrm{t}+1}^{2}=\omega+\alpha \sigma_{\mathrm{t}}^{2}\left(((\mathrm{~d}-2) / \mathrm{d}) \mathrm{z}_{\mathrm{t}}-\theta\right)^{2}+\beta \sigma_{\mathrm{t}}^{2}
\end{aligned}
$$

where $R_{t+1}$ is the daily demeaned $\mathrm{P} / \mathrm{L}$ and the innovations $z_{t}$ are drawn independently from a Student's $\mathrm{t}(\mathrm{d})$ distribution. The Student-t innovations enable the model to capture some of the additional kurtosis.

Table 3 reports the maximum likelihood estimates from the GARCH model for each business line. As usual we get a small but positive $\alpha$ and a $\beta$ much closer to 1 . Variance persistence in this model is given by $\alpha\left(1+\theta^{2}\right)+\beta$. It is largest in business lines 2 and 4 which confirm the impression provided by Figure 2. The last three lines of Table 3 report the log likelihood values for the four GARCH models along with the log likelihood values for the case of no variance dynamics, where $\alpha=\beta=\theta=0$.

Looking across the four GARCH estimates we see that Desk 1 is characterized by a large $\alpha$ and small $\mathrm{d}$ which suggests are large conditional kurtosis. Desk 2 is 
characterized by high variance persistence and high unconditional kurtosis from the low d. Desk 3 has an unusually large negative $\theta$ which suggests that a positive $\mathrm{P} / \mathrm{L}$ increases volatility by more than a negative $\mathrm{P} / \mathrm{L}$ of the same magnitude. Desk 4 has an unusually large unconditional volatility and a relatively high persistence as noted earlier.

For the power simulation exercise, we will assume that the correct data-generating processes are the four estimated GARCH processes. We must also choose a particular implementation for the VaR calculation. Following the approach used by the bank to construct their internal VaR forecasts, we make use of historical simulation or "bootstrapping". The historical simulation VaR on a certain day is simply the unconditional quantile of the past $T_{e}$ daily observations. Specifically

$$
\operatorname{VaR}_{t+1}^{p}=- \text { percentile }\left(\left\{R_{s}\right\}_{s=t-T_{e}+1}^{t}, 100 p\right)
$$

For the purposes of this Monte Carlo experiment, we choose $T_{e}=250$ corresponding to 250 trading days. The VaR coverage rate $p$ we study is either 1\% (as in Section 2) or 5\%. We look at one-day ahead VaR again as in Section 2. When computing the finite-sample p-values we use $N=9,999$ and we perform 10,000 simulations for each test.

Table 4 shows the finite sample power results for the $1 \%$ VaR from Historical Simulation for various samples sizes when using the GARCH DGP processes corresponding to each of the four business lines.

For business line 1 it appears that the $\mathrm{LB}(5)$ test performs the best overall, although the Geometric, KS and CVM tests are slightly better for the smallest sample. For the largest sample the Caviar test performs the best. For business line 2 the Geometric test performs the best in all cases with the Caviar a close second in the largest sample. For business line 3 the power is low everywhere and the Caviar is best overall. For business line 4 the Geometric test is again best everywhere.

Consider next Table 5 which shows reports the finite sample power calculations for the $5 \% \mathrm{VaR}$. For business line 1 the Caviar is best over all with the LB(5) slightly better in a couple of cases. For business line 2 the Caviar test is best for small samples and the Geometric best for larger samples. For business line 3 the power is again low 
everywhere and the Caviar test performs the best. For business line 4 the Caviar is again best for small samples and the Geometric is best for large samples.

Considering Table 4 and 5 overall it appears that the Caviar test is the best for 5\% $\mathrm{VaR}$ and the Geometric is best for $1 \% \mathrm{VaR}$. For business line 3 the results are somewhat surprising and require further investigation.

Tables 4 and 5 provide a couple of other conclusions. First, it is clear that the $\mathrm{LB}(5)$ test is better than $\mathrm{LB}(1)$ and Markov test. This is perhaps to be expected as the dependence in the hit sequence is not of order 1 here. Second, the Geometric test is typically substantially better than the Weibull test. This is also to be expected as the latter wrongly assumes a continuous distribution for the duration variable.

Overall the power of the best tests investigated here is quite impressive, particularly considering the small samples investigated. Admittedly, the power numbers reported in Tables 4 and 5 are affected by the fact that we have conditioned the power calculations on being able to calculate the test in the first place. Samples where the tests cannot be computed are omitted due to lack of VaR hits.

\section{Feasibility Ratios}

For transparency we report in Table 6 the fraction of simulated samples from Tables 4 and 5 where the each test is feasible. We only report sample sizes 250,500 , and 750 for the $1 \% \mathrm{VaR}$ and 250 for the $5 \% \mathrm{VaR}$ as the other sample sizes had no omitted sample paths in our experiment. Table 4 shows that only in the case of $1 \% \mathrm{VaR}$ and samples of 250 observations is the issue non-trivial. In those cases the issue is most serious for the Weibull and Geometric tests and least serious for the Caviar test. That conclusion also holds when considering the bottom panel in Figure 6 which reports the fraction of feasible samples from the size calculations in Table 2.

\section{Results for Desk-level Data}

In Table 7 we report the results from applying our tests to the actual observed sequences of $\mathrm{P} / \mathrm{Ls}$ and VaRs from the four business lines. As in the power calculations 
above we make use of the Dufour (2004) Monte Carlo testing technique which yields tests with correct level, regardless of sample size.

For the case of a continuous test statistic, the procedure is the following. We first generate $N$ independent realizations of the test statistic, $L R_{i}, i=1, \ldots, N$. We denote by $L R_{0}$ the test statistic computed with the original sample. Under the hypothesis that the risk model is correct, we know that the hit sequence is i.i.d. Bernoulli with the mean equal to the coverage rate. We thus benefit from the advantage of not having nuisance parameters under the null hypothesis.

We next rank $L R_{i}, i=0,1, \ldots, N$ in non-decreasing order and obtain the Monte Carlo p-value $\hat{p}_{N}\left(L R_{0}\right)$, where

$$
\hat{p}_{N}\left(L R_{0}\right)=\frac{N \hat{G}\left(L R_{0}\right)+1}{N+1}
$$

and

$$
\hat{G}_{N}\left(L R_{0}\right)=\frac{1}{N} \sum_{i=1}^{N} \mathrm{I}\left(L R_{i}>L R_{0}\right)
$$

The indicator function $\mathrm{I}(\cdot)$ takes on the value one if true and the value zero otherwise. We reject the null hypothesis if $\hat{p}_{N}\left(L R_{0}\right)$ is less or equal than the prespecified significance level.

When working with binary sequences, there is a non-zero probability of obtaining ties between the test values obtained with the sample and the simulated data. The tiebreaking procedure is as follows: For each test statistic, $L R_{i}, i=0,1, \ldots, N$, we draw an independent realization of a uniform distribution on the $[0 ; 1]$ interval. Denote these draws by $U_{i}, i=0,1, \ldots, N$. We obtain the Monte Carlo p-value by replacing $\hat{\mathrm{G}}_{\mathrm{N}}\left(\mathrm{LR}_{0}\right)$ with

$$
\tilde{G}_{N}\left(L R_{0}\right)=1-\frac{1}{N} \sum_{i=1}^{N} \mathrm{I}\left(L R_{i} \leq L R_{0}\right)+\frac{1}{N} \sum_{i=1}^{N} \mathrm{I}\left(L R_{i}=L R_{0}\right) \mathrm{I}\left(U_{i} \geq U_{0}\right)
$$

There are two additional advantages of using a simulation procedure. The first is that possible systematic biases, for example arising from the use of a continuous distribution to study discrete processes, are accounted for since they will appear both in 
$L R_{0}$ and $L R_{i}$. The second is that Monte Carlo testing procedures are consistent even if the parameter value is on the boundary of the parameter space. The bootstrap procedures on the other hand could be inconsistent in this case.

In Table 7 we report the results from applying our tests to the actual observed sequences of $\mathrm{P} / \mathrm{Ls}$ and VaRs from the four business lines. We find no rejections in the first two business lines and multiple rejections in business line 3. In business line 4 two of the tests reject the risk model. Thus, when backtesting the actual VaRs from the four business unit we find it difficult to reject the historical simulation VaR except for business line 3. This finding could of course have several explanations. First, the samples are short. Second, random chance, that is, by design we fail to reject $10 \%$ of the time. Third, the bank may be implementing historical simulation with some adjustments which make it difficult to reject by the tests considered here.

\section{Conclusions}

With the introduction of RiskMetrics, JP Morgan (1994) sparked a revolution in the field of market risk management. RiskMetrics has many redeeming features such as dynamic volatility and correlation modeled in a very parsimonious fashion.

However other aspects such as the conditional normality assumption, and the difficulty of aggregating VaRs across business lines has increased the popularity of the model-free Historical Simulation technique in the financial services industry. Commercial banks in particular have increasingly trended towards the use of Historical Simulation for market risk management.

Using new desk-level P/Ls and VaRs data from four business lines in a large international commercial bank we discuss the pros and cons of this trend. We find strong evidence of volatility dynamics and non-normality in daily $\mathrm{P} / \mathrm{Ls}$. Volatility dynamics are not captured in HistSim and may therefore cause clustering in VaR violations which can have important economic effects such as increased risk of default. We assess the ability of external bank regulators and internal risk auditors to detect problems in HistSim-based VaRs using a wide range of existing and new backtesting procedures.

The relatively sluggish dynamics of HistSim is often touted as a virtue in that it avoids frequent adjustments to the associated risk-based capital. However, the internal 
desk-level VaRs we present here move quite rapidly at the daily level thus casting doubt on the common wisdom. The extreme spikes in P/Ls particularly evident in lines 1 and 2 highlight the dangers of relying on the normal distribution in market risk management. Fortunately, the HistSim VaRs do not rely on the normal distribution, which is clearly one of its redeeming features.

Larger backtesting sample sizes would clearly be helpful. Second, aggregate GARCH modeling may be desirably to remove clusters in the VaR violations. Third, ideally the historical dataset of asset prices should be updated daily so as to capture current volatility trends. Fourth, simultaneous reporting of the VaR with several coverage rates (e.g. $1 \%, 2.5 \%, 5 \%)$ would be helpful to assess more carefully the tail distribution of the $\mathrm{P} / \mathrm{L}$ and to detect omitted variance dynamics. 


\section{References}

Artzner, P., F. Delbaen, J. Eber, and D. Heath (1999), "Coherent Measures of Risk," Mathematical Finance, 9, 203-228.

Basak, S. and A. Shapiro (2001), "Value-at-Risk Based Risk Management: Optimal Policies and Asset Prices," Review of Financial Studies, 14, 371-405.

Basle Committee on Banking Supervision (1996), Amendment to the Capital Accord to Incorporate Market Risks.

Basle Committee on Banking Supervision (2004), International Convergence of Capital Measurement and Capital Standards: a Revised Framework.

Berkowitz, J. (2001), “Generalized Spectral Estimation of the Consumption-Based Asset Pricing Model," Journal of Econometrics, 104, 269-288.

Berkowitz, J. and J. O'Brien (2002), "How Accurate are the Value-at-Risk Models at Commercial Banks?” Journal of Finance, 57, 1093-1111.

Campbell, John Y., and Robert J. Shiller (1987), "Cointegration and Tests of Present Value Models", Journal of Political Economy, 95, 1062-1088.

Christoffersen, Peter F. (1998), "Evaluating interval forecasts," International Economic Review 39, 841-862.

Christoffersen, Peter F., and Silvia Goncalves (2005), "Estimation Risk in Financial Risk Management, Journal of Risk, 7, 1-28.

Christoffersen, Peter F. and Denis Pelletier (2004), "Backtesting Value-at-Risk: A Duration-Based Approach," Journal of Financial Econometrics, 2, 84-108.

Cuoco, D., H. He and S. Issaenko (2001), "Optimal Dynamic Trading Strategies with Risk Limits," Manuscript, The Wharton School, University of Pennsylvania.

Diebold, Francis X., Todd A. Gunther, and Anthony S. Tay (1998), "Evaluating density forecasts," International Economic Review 39, 863-883.

Dufour, Jean-Marie, (2004), "Monte Carlo Tests with Nuisance Parameters: A General Approach to Finite-Sample Inference and Nonstandard Asymptotics", Manuscript, University of Montreal. Forthcoming in the Journal of Econometrics.

Durlauf, S. N. (1991), "Spectral Based Testing of the Martingale Hypothesis," Journal of Econometrics, 50, 355-376. 
Engle, Robert F. and Simone Manganelli (2004), "CAViaR: Conditional autoregressive Value-at-Risk by regression quantiles," Journal of Business and Economic Statistics, 22, 367-381.

Engle, Robert F. and Jeffrey Russel (1998), “Autoregressive Conditional Duration: A New Model for Irregularly Spaced Transaction Data,” Econometrica, 66, 11271162.

Gourieroux, C. (2000). Econometrics of Qualitative Dependent Variables. Translated by Paul B. Klassen. Cambridge University Press.

Harrison, M. and D. Kreps (1979), "Martingales and Arbitrage in Multi-period Securities Markets," Journal of Economic Theory, 20, 381-408.

Harrison, M., and S. Pliska (1981), "Martingales and Stochastic Integrals," in the Theory of Continuous Trading, Stochastic Processes and their Applications, 11, 215-260.

Hendricks, Darryl (1996), "Evaluation of Value-at-Risk models using historical data," Economic Policy Review, Federal Reserve Bank of New York, April, 39-69.

Jorion, Philippe (2001). Value-at-Risk: the New Benchmark for Controlling Market Risk McGraw-Hill: Chicago.

Jorion, Philippe (2002), "How informative are Value-at-Risk disclosures," Accounting Review, 77, 911-931.

JP Morgan (1994), "RiskMetrics,” Technical Document. New York.

Kiefer, N. (1988). "Economic Duration Data and Hazard Functions." Journal of Economic Literature, 26, 646-679.

Kupiec, P. (1995), “Techniques for Verifying the Accuracy of Risk Measurement Models," Journal of Derivatives, 3, 73-84. 


\section{Figure 1: P/Ls and VaRs for Four Business Lines}
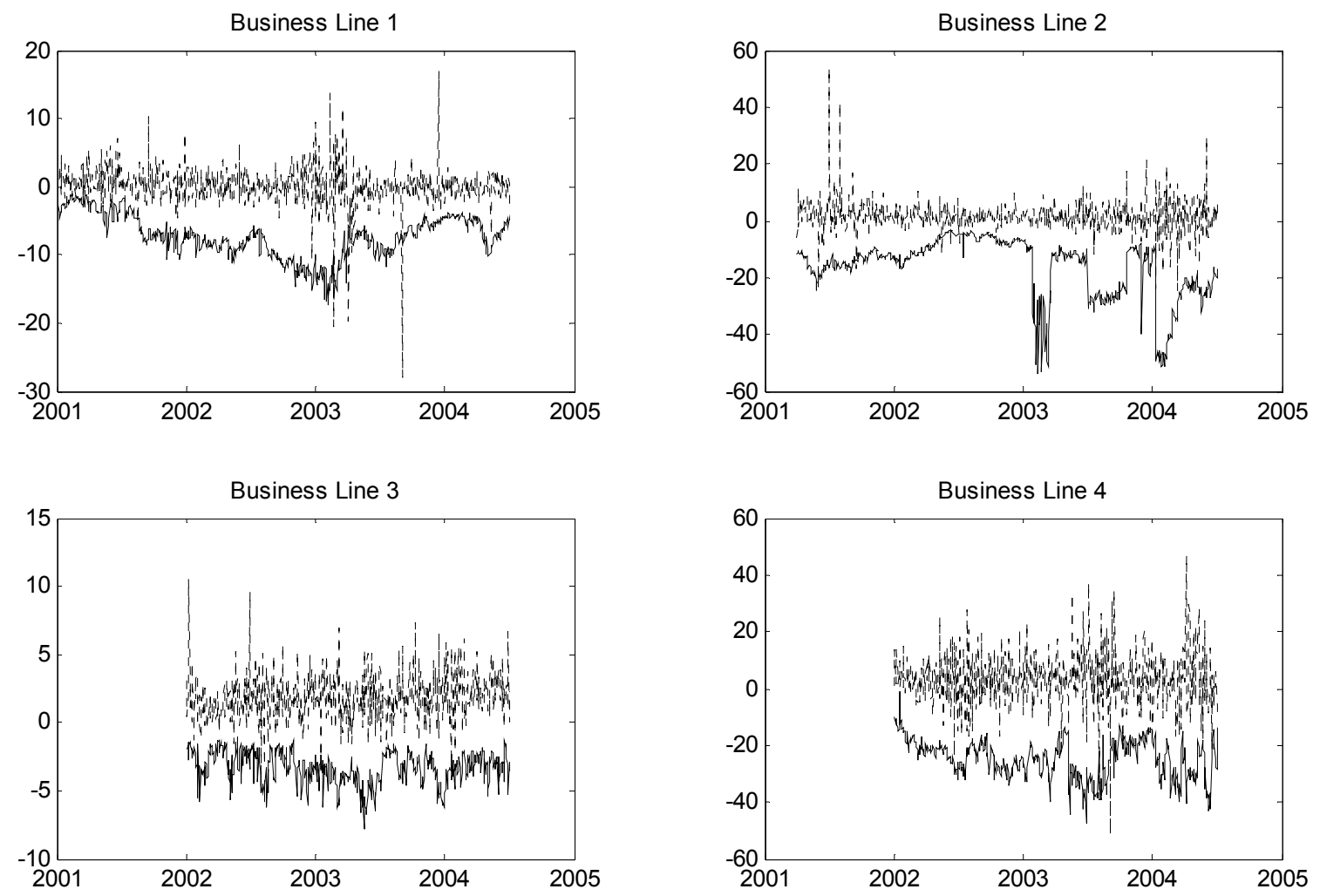

Notes to Figure: We plot the P/Ls (dashed lines) and VaRs (solid lines) from the four business lines. 
Figure 2: Absolute Demeaned P/Ls for Four Business Lines
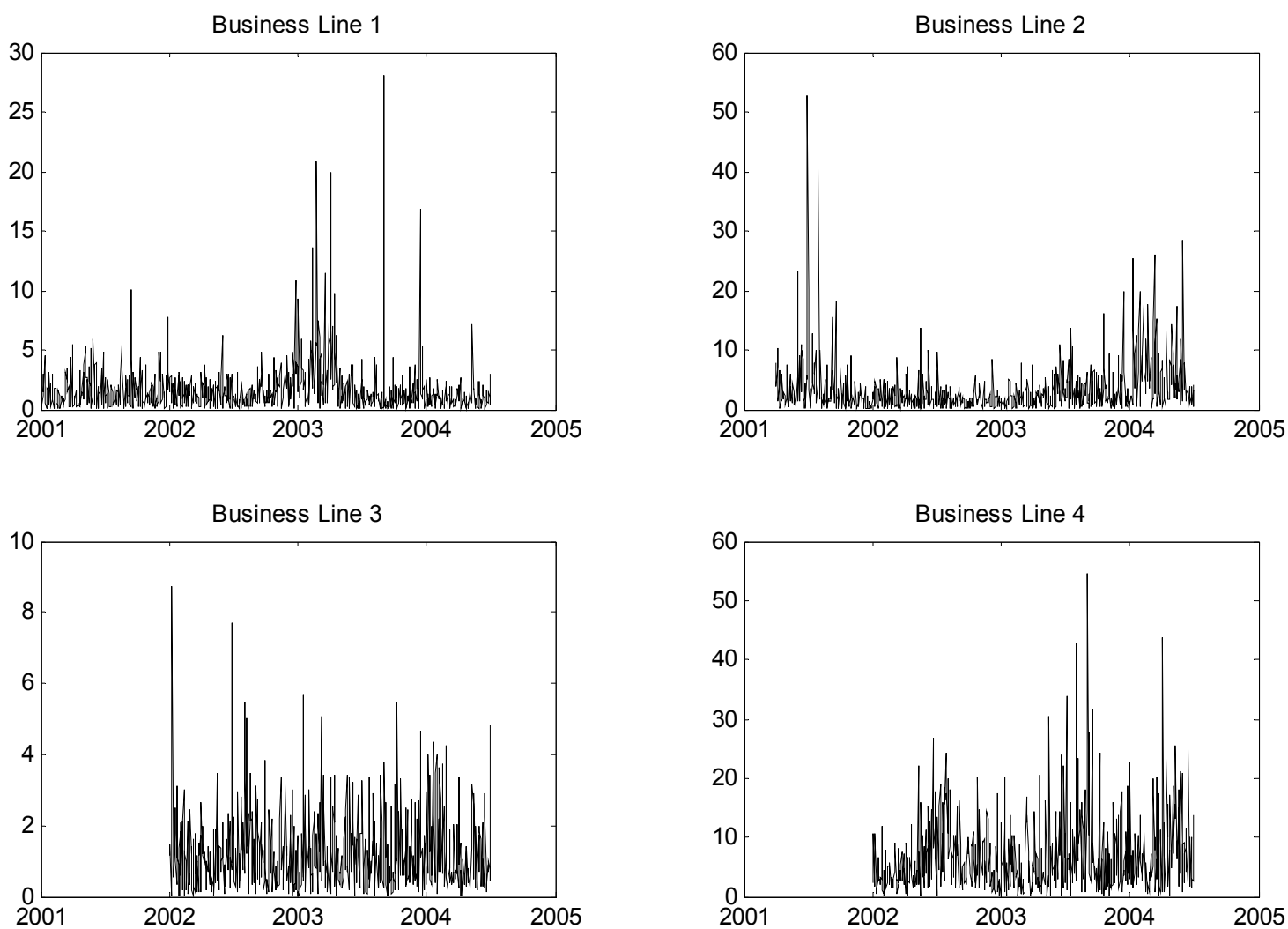

Notes to Figure: We subtract the sample mean from each of the four P/Ls in Figure 1 and plot the absolute value of these centered P/Ls. 
Table 1: P/Ls and VaRs for Four Business Lines: Descriptive Statistics

\begin{tabular}{|c|c|c|c|c|}
\hline & \multicolumn{4}{|c|}{ P/Ls } \\
\hline & $\underline{\text { Desk } 1}$ & Desk 2 & Desk 3 & Desk 4 \\
\hline Number of Observations & 873 & 811 & 623 & 623 \\
\hline Mean & 0.1922 & 1.5578 & 1.8740 & 3.1562 \\
\hline Standard Deviation & 2.6777 & 5.2536 & 1.6706 & 9.2443 \\
\hline Skewness & -1.7118 & 1.5441 & 0.5091 & -0.1456 \\
\hline \multirow[t]{3}{*}{ Excess Kurtosis } & 24.2195 & 19.8604 & 2.0060 & 3.6882 \\
\hline & \multicolumn{4}{|c|}{ VaRs } \\
\hline & $\underline{\text { Desk } 1}$ & $\underline{\text { Desk } 2}$ & Desk 3 & Desk 4 \\
\hline Number of Observations & 873 & 811 & 623 & 623 \\
\hline Mean & -7.2822 & -16.3449 & -3.2922 & -24.8487 \\
\hline Standard Deviation & 3.1321 & 10.5446 & 1.1901 & 6.6729 \\
\hline Skewness & -0.3038 & -1.3746 & -0.6529 & -0.3006 \\
\hline Kurtosis & -0.1525 & 1.6714 & -0.0133 & -0.1211 \\
\hline Observed Number of Hits & 9 & 5 & 1 & 4 \\
\hline Expected Number of Hits & 9 & 8 & 6 & 6 \\
\hline
\end{tabular}


Table 2: Size of 10\% Asymptotic CC Tests

\begin{tabular}{|c|c|c|c|c|c|c|c|c|}
\hline \multicolumn{9}{|c|}{$1 \%$ VaR } \\
\hline Sample & $\underline{\mathrm{LB}(1)}$ & $\underline{\mathrm{LB}(5)}$ & Markov & Weibull & Geometric & Caviar & $\underline{\mathrm{KS}}$ & CVM \\
\hline 250 & 0.0253 & 0.0999 & 0.0497 & 0.1103 & 0.5306 & 0.0505 & 0.0390 & 0.0517 \\
\hline 500 & 0.0440 & 0.1336 & 0.0676 & 0.1759 & 0.2332 & 0.1153 & 0.0664 & 0.1120 \\
\hline 750 & 0.0669 & 0.1650 & 0.0663 & 0.1616 & 0.1582 & 0.1426 & 0.0923 & 0.1241 \\
\hline 1000 & 0.0763 & 0.1465 & 0.0759 & 0.1569 & 0.1186 & 0.1207 & 0.0944 & 0.1247 \\
\hline 1250 & 0.1022 & 0.1458 & 0.05 & 0.1276 & 0.11 & 0.1214 & 0.1120 & 0.1401 \\
\hline 1500 & 0.1005 & 0.1309 & 0.0637 & 0.1273 & 0.0954 & 0.1116 & 0.1117 & 0.1372 \\
\hline \multicolumn{9}{|c|}{$5 \% \mathrm{VaR}$} \\
\hline Sample & $\underline{\mathrm{LB}(1)}$ & $\underline{\mathrm{LB}(5)}$ & Markov & Weibull & Geometric & Caviar & $\underline{\mathrm{KS}}$ & CVM \\
\hline 250 & 0.0805 & 0.1080 & 0.1280 & 0.1336 & 0.0976 & 0.1053 & $0 . \overline{1020}$ & 0.1056 \\
\hline 500 & 0.0675 & 0.1009 & 0.1284 & 0.1252 & 0.0762 & 0.1010 & 0.0907 & 0.0830 \\
\hline 750 & 0.0685 & 0.1018 & 0.1659 & 0.1400 & 0.0678 & 0.0964 & 0.0965 & 0.0863 \\
\hline 1000 & 0.0891 & 0.0965 & 0.2085 & 0.1423 & 0.0718 & 0.0970 & 0.0951 & 0.0950 \\
\hline 1250 & 0.0920 & 0.0925 & 0.1607 & 0.1490 & 0.0608 & 0.0954 & 0.0956 & 0.0982 \\
\hline 1500 & 0.0866 & 0.0978 & 0.1515 & 0.1596 & 0.0630 & 0.1029 & 0.0949 & 0.0974 \\
\hline
\end{tabular}


Table 3: P/L GARCH Model Parameters and Properties

\begin{tabular}{|c|c|c|c|c|}
\hline & $\underline{\text { Desk } 1}$ & Desk 2 & Desk 3 & Desk 4 \\
\hline $\mathrm{d}$ & 3.808 & 3.3183 & 6.9117 & 4.7017 \\
\hline$\theta$ & -0.245 & 0.5031 & -0.9616 & 0.0928 \\
\hline$\beta$ & 0.7495 & 0.9284 & 0.8728 & 0.9153 \\
\hline$\alpha$ & 0.1552 & 0.0524 & 0.0261 & 0.0723 \\
\hline$\omega$ & 0.5469 & 0.2154 & 0.2127 & 1.6532 \\
\hline Variance Persistence & 0.9140 & 0.9941 & 0.9230 & 0.9882 \\
\hline Unconditional Stdev & 2.5220 & 6.0233 & 1.6624 & 11.8478 \\
\hline $\operatorname{LogL}$ & -1360.76 & -1781.25 & -825.87 & -1855.98 \\
\hline LogL (HomoSked.) & -1401.64 & -1843.49 & -831.46 & -1877.73 \\
\hline P-value & 0.0000 & 0.0000 & 0.0108 & 0.0000 \\
\hline
\end{tabular}


Table 4: Power of 10\% Finite Sample CC Tests on 1\% VaR in Four Business Lines

\begin{tabular}{|c|c|c|c|c|c|c|c|c|}
\hline \multicolumn{9}{|c|}{ Business Line 1} \\
\hline Sample & $\underline{\mathrm{LB}(1)}$ & $\underline{\mathrm{LB}(5)}$ & Markov & Weibull & $\underline{\text { Geometric }}$ & $\underline{\text { Caviar }}$ & $\underline{\mathrm{KS}}$ & $\underline{\text { CVM }}$ \\
\hline 250 & 0.1958 & $\overline{0.3201}$ & 0.1862 & 0.1431 & 0.3258 & $\overline{0.1340}$ & $0 . \overline{3273}$ & $\overline{0.3305}$ \\
\hline 500 & 0.2293 & 0.4200 & 0.1914 & 0.1439 & 0.2642 & 0.3360 & 0.4112 & 0.3563 \\
\hline 750 & 0.2997 & 0.4755 & 0.1903 & 0.1474 & 0.2758 & 0.4090 & 0.4607 & 0.4075 \\
\hline 1000 & 0.3709 & 0.5185 & 0.1678 & 0.1820 & 0.3418 & 0.4810 & 0.5083 & 0.4725 \\
\hline 1250 & 0.4335 & 0.5635 & 0.1874 & 0.2280 & 0.3704 & 0.5360 & 0.5421 & 0.5027 \\
\hline 1500 & 0.4463 & 0.6027 & 0.2018 & 0.2444 & 0.4098 & 0.6500 & 0.5852 & 0.5638 \\
\hline
\end{tabular}

Business Line 2

\begin{tabular}{|c|c|c|c|c|c|c|c|c|}
\hline ample & $\underline{\mathrm{LB}(1)}$ & $\underline{\mathrm{LB}(5)}$ & Markov & Weibull & $\underline{\text { Geometric }}$ & $\underline{\text { Caviar }}$ & $\underline{\mathrm{KS}}$ & $\underline{\text { CVM }}$ \\
\hline 250 & 0.2312 & 0.2320 & 0.2110 & 0.1373 & 0.3654 & 0.1420 & 0.2777 & 0.2662 \\
\hline 500 & 2201 & 0.2960 & 0.1896 & 0.15 & 0.3 & & 0.3146 & 0.2689 \\
\hline 750 & 0.2372 & 0.3319 & 0.1806 & 0.1795 & 0.3866 & 0.3 & 0.3407 & 0.2810 \\
\hline 1000 & 0.2806 & 0.3614 & 0.1726 & 0.2181 & 0.4214 & 0.3750 & 0.3895 & 0.3229 \\
\hline 1250 & 0.2808 & 0.4001 & 0.1604 & 0.2652 & & & 0.3807 & 0.3265 \\
\hline 1500 & 0.2795 & 0.4231 & 0.1604 & 0.3041 & 0.5068 & 0.4830 & 0.4263 & 0.3707 \\
\hline
\end{tabular}

Business Line 3

\begin{tabular}{|c|c|c|c|c|c|c|c|c|}
\hline Sample & $\underline{\mathrm{LB}(1)}$ & $\underline{\mathrm{LB}(5)}$ & $\underline{\text { Markov }}$ & $\underline{\text { Weibull }}$ & $\underline{\text { Geometric }}$ & $\underline{\text { Caviar }}$ & $\underline{\mathrm{KS}}$ & $\underline{\text { CVM }}$ \\
\hline 250 & 0.0769 & 0.1170 & 0.0725 & 0.0794 & 0.1372 & 0.1020 & 0.1127 & 0.1158 \\
\hline 500 & 0.0677 & 0.1532 & 0.0625 & 0.07 & & 0.2 & 0.1276 & 0.1078 \\
\hline 750 & 0.0899 & 0.1603 & 0.0529 & 0.05 & 0.05 & 0.2 & 0.1264 & 0.1122 \\
\hline 1000 & 0.1059 & 0.1462 & 0.0359 & 0.0507 & 0.04 & 0.32 & 0.1374 & 0.1209 \\
\hline 1250 & 0.1305 & 0.1273 & 0.0390 & 0.0493 & & 0.4060 & 0.1373 & 0.1295 \\
\hline 1500 & 0.1472 & 0.1260 & 0.0311 & 0.0421 & 0.0380 & 0.5470 & 0.1498 & 0.1473 \\
\hline
\end{tabular}

Business Line 4

\begin{tabular}{|c|c|c|c|c|c|c|c|c|}
\hline Sample & $\underline{\mathrm{LB}(1)}$ & $\underline{\mathrm{LB}(5)}$ & Markov & $\underline{\text { Weibull }}$ & $\underline{\text { Geometric }}$ & $\underline{\text { Caviar }}$ & $\underline{\mathrm{KS}}$ & $\underline{\text { CVM }}$ \\
\hline 250 & 0.2502 & 0.2637 & 0.2338 & 0.1593 & 0.4058 & 0.1720 & 0.3132 & 0.3016 \\
\hline 500 & 0.2400 & 0.3367 & 0.2142 & 0.18 & & 0.3 & 0.3824 & 0.2984 \\
\hline 750 & 0.2803 & 0.3824 & 0.2038 & 0.2118 & 0.4288 & 0.3 & 0.4030 & 0.3216 \\
\hline 1000 & 0.3326 & 0.4193 & 0.2019 & 0.2671 & 0.5026 & 0.4350 & 0.4494 & 0.3753 \\
\hline 1250 & 0.3170 & 0.4580 & 0.1955 & 0.3432 & & 0.4960 & 0.4546 & 0.3919 \\
\hline 1500 & 0.3293 & 0.5095 & 0.2018 & 0.3889 & 0.5968 & 0.5630 & 0.4881 & 0.4272 \\
\hline
\end{tabular}


Table 5: Power of 10\% Finite Sample CC Tests on 5\% VaR in Four Business Lines

\begin{tabular}{|c|c|c|c|c|c|c|c|c|}
\hline \multicolumn{9}{|c|}{ Business Line 1} \\
\hline$\underline{\text { Sample }}$ & $\underline{\mathrm{LB}(1)}$ & $\underline{\mathrm{LB}(5)}$ & Markov & $\underline{\text { Weibull }}$ & $\underline{\text { Geometric }}$ & $\underline{\text { Caviar }}$ & $\underline{\mathrm{KS}}$ & $\underline{\text { CVM }}$ \\
\hline 250 & 0.2964 & 0.3852 & 0.2048 & 0.1613 & 0.3190 & $\overline{0.4240}$ & $0 . \overline{3485}$ & 0.3442 \\
\hline 500 & 0.3912 & 0.5275 & 0.2139 & 0.1825 & 0.4466 & 0.5100 & 0.4429 & 0.4638 \\
\hline 750 & 0.4356 & 0.6334 & 0.2257 & 0.2305 & 0.5684 & 0.6240 & 0.5316 & 0.5534 \\
\hline 1000 & 0.4836 & 0.6957 & 0.2511 & 0.2698 & 0.6794 & 0.7290 & 0.5858 & 0.6068 \\
\hline 1250 & 0.5431 & 0.7621 & 0.2935 & 0.3246 & 0.7560 & 0.7940 & 0.6654 & 0.6745 \\
\hline 1500 & 0.5925 & 0.8146 & 0.3279 & 0.3790 & 0.8188 & 0.8590 & 0.7199 & 0.7220 \\
\hline
\end{tabular}

Business Line 2

\begin{tabular}{|c|c|c|c|c|c|c|c|c|}
\hline ample & $\underline{\mathrm{LB}(1)}$ & $\underline{\mathrm{LB}(5)}$ & Markov & Weibull & $\underline{\text { Geometric }}$ & $\underline{\text { Caviar }}$ & $\underline{\mathrm{KS}}$ & $\underline{\text { CVM }}$ \\
\hline 250 & 0.2592 & 0.3575 & 0.3404 & 0.3222 & 0.4222 & 0.5630 & 0.3901 & 0.3832 \\
\hline 500 & 3421 & 0.5077 & 0.2978 & 0.3 & & 0.6 & 0.4482 & 0.4492 \\
\hline 750 & 0.3757 & 0.5972 & 0.2719 & 0.4 & & 0.6 & 0.5041 & 0.5059 \\
\hline 1000 & 0.4194 & 0.6581 & 0.2759 & 0.4869 & 0.7838 & 0.7140 & 0.5584 & 0.5493 \\
\hline 1250 & 0.4663 & 0.7208 & 0.3103 & & & & 0.6247 & 0.6090 \\
\hline 1500 & 0.5038 & 0.7806 & 0.3345 & 0.6056 & 0.8996 & 0.8340 & 0.6811 & 0.6554 \\
\hline
\end{tabular}

Business Line 3

\begin{tabular}{|c|c|c|c|c|c|c|c|c|}
\hline Sample & $\underline{\mathrm{LB}(1)}$ & $\underline{\mathrm{LB}(5)}$ & Markov & $\underline{\text { Weibull }}$ & $\underline{\text { Geometric }}$ & $\underline{\text { Caviar }}$ & $\underline{\mathrm{KS}}$ & $\underline{\mathrm{CVM}}$ \\
\hline 250 & 0.1080 & 0.1129 & 0.0821 & 0.0676 & 0.0888 & 0.2260 & 0.0986 & 0.0991 \\
\hline 500 & 0.1032 & 0.1199 & 0.0653 & 0.0403 & 0.06 & 0.3390 & 0.1050 & 0.1122 \\
\hline 750 & 0.1032 & 0.1246 & 0.0617 & 0.0343 & 0.0490 & 0.4220 & 0.1063 & 0.1160 \\
\hline 1000 & 0.1126 & 0.1225 & 0.0624 & 0.0310 & 0.0524 & 0.5200 & 0.1021 & 0.1069 \\
\hline 1250 & 0.1140 & 0.1251 & 0.0686 & 0.0285 & & & 0.1131 & 0.1216 \\
\hline 1500 & 0.1071 & 0.1247 & 0.0653 & 0.0333 & 0.0534 & 0.7160 & 0.1171 & 0.1163 \\
\hline
\end{tabular}

Business Line 4

\begin{tabular}{|c|c|c|c|c|c|c|c|c|}
\hline Sample & $\underline{\mathrm{LB}(1)}$ & $\underline{\mathrm{LB}(5)}$ & Markov & $\underline{\text { Weibull }}$ & $\underline{\text { Geometric }}$ & $\underline{\text { Caviar }}$ & $\underline{\mathrm{KS}}$ & $\underline{\text { CVM }}$ \\
\hline 250 & 0.2877 & 0.3926 & 0.3305 & 0.3260 & 0.4460 & 0.5400 & 0.4089 & 0.3926 \\
\hline 500 & 0.3531 & 0.5360 & 0.2816 & & & & 0.4704 & 0.4743 \\
\hline 750 & 0.3976 & 0.6367 & 0.2666 & 0.4652 & 0.7462 & 0.7000 & 0.5453 & 0.5397 \\
\hline 1000 & 0.4433 & 0.7050 & 0.2779 & 0.5401 & 0.8 & 0.7430 & 0.6059 & 0.5917 \\
\hline 1250 & 0.5010 & 0.7694 & 0.3169 & 0.6128 & & 0.7990 & 0.6670 & 0.6583 \\
\hline 1500 & 0.5482 & 0.8239 & 0.3609 & 0.6773 & 0.9356 & 0.8520 & 0.7340 & 0.7130 \\
\hline
\end{tabular}


Table 6: Fraction of Samples where Test is Feasible. 1\% and 5\% VaR

\begin{tabular}{ccccccccccc}
\multicolumn{10}{c}{ Power Simulation: Business Line 1 } \\
$\frac{\text { VaR }}{1 \%}$ & $\underline{\text { Sample }}$ & & $\underline{\mathrm{LB}(1)}$ & $\underline{\mathrm{LB}(5)}$ & $\underline{\text { Markov }}$ & $\underline{\text { Weibull }}$ & $\underline{\text { Geometric }}$ & $\underline{\text { Caviar }}$ & $\underline{\mathrm{KS}}$ & $\underline{\mathrm{CVM}}$ \\
$1 \%$ & 500 & 0.9081 & 0.9081 & 0.9006 & 0.6974 & 0.8322 & 0.9897 & 0.9081 & 0.9081 \\
$1 \%$ & 750 & 1.0000 & 1.0000 & 1.0000 & 0.9998 & 0.9999 & 1.0000 & 0.9999 & 1.0000 \\
\hline $5 \%$ & 250 & 0.9998 & 0.9998 & 0.9998 & 0.9984 & 1.0000 & 1.0000 & 0.9999 & 1.0000
\end{tabular}

\begin{tabular}{ccccccccccc}
\multicolumn{10}{c}{ Power Simulation: Business Line 2 } \\
$\underline{\underline{V} \text { VaR }}$ & $\underline{\text { Sample }}$ & $\underline{\underline{\mathrm{LB}}(1)}$ & $\underline{\mathrm{LB}(5)}$ & $\underline{\text { Markov }}$ & $\underline{\underline{\text { Weibull }}}$ & $\underline{\text { Geometric }}$ & $\underline{\text { Caviar }}$ & $\underline{\text { KS }}$ & $\underline{\underline{\mathrm{CVM}}}$ \\
$1 \%$ & 250 & 0.8693 & 0.8693 & 0.8643 & 0.6691 & 0.8167 & 0.9844 & 0.8693 & 0.8693 \\
$1 \%$ & 500 & 0.9916 & 0.9916 & 0.9928 & 0.9654 & 0.9824 & 0.9997 & 0.9927 & 0.9929 \\
$1 \%$ & 750 & 0.9996 & 0.9996 & 0.9999 & 0.9986 & 0.9996 & 0.9999 & 0.9997 & 0.9997 \\
\hline $5 \%$ & 250 & 0.9965 & 0.9965 & 0.9949 & 0.9881 & 0.9942 & 0.9994 & 0.9963 & 0.9973
\end{tabular}

\begin{tabular}{ccccccccccc}
\multicolumn{10}{c}{ Power Simulation: Business Line 3 } \\
$\underline{\underline{V a R}}$ & $\underline{\text { Sample }}$ & $\underline{\underline{\mathrm{LB}}(1)}$ & $\underline{\mathrm{LB}(5)}$ & $\underline{\text { Markov }}$ & $\underline{\underline{\text { Weibull }}}$ & $\underline{\text { Geometric }}$ & $\underline{\text { Caviar }}$ & $\underline{\mathrm{KS}}$ & $\underline{\mathrm{CVM}}$ \\
$1 \%$ & 250 & 0.9356 & 0.9356 & 0.9371 & 0.7077 & 0.8477 & 0.9945 & 0.9356 & 0.9356 \\
$1 \%$ & 500 & 0.9990 & 0.9990 & 0.9998 & 0.9916 & 0.9943 & 1.0000 & 0.9990 & 0.9990 \\
$1 \%$ & 750 & 1.0000 & 1.0000 & 1.0000 & 0.9999 & 0.9999 & 1.0000 & 1.0000 & 1.0000 \\
\hline $5 \%$ & 250 & 1.0000 & 1.0000 & 1.0000 & 1.0000 & 1.0000 & 1.0000 & 1.0000 & 1.0000
\end{tabular}

\begin{tabular}{|c|c|c|c|c|c|c|c|c|c|}
\hline \multicolumn{10}{|c|}{ Power Simulation: Business Line 4} \\
\hline$\underline{\mathrm{VaR}}$ & $\underline{\text { Sample }}$ & $\underline{\mathrm{LB}(1)}$ & $\underline{\mathrm{LB}(5)}$ & Markov & Weibull & $\underline{\text { Geometric }}$ & $\underline{\text { Caviar }}$ & $\underline{\mathrm{KS}}$ & $\underline{\text { CVM }}$ \\
\hline $1 \%$ & 250 & $\overline{0.8659}$ & 0.8659 & 0.8660 & 0.6775 & 0.8169 & 0.9829 & 0.8659 & 0.8659 \\
\hline $1 \%$ & 500 & 0.9935 & 0.9935 & 0.9940 & 0.9694 & 0.9839 & 1.0000 & 0.9941 & 0.9946 \\
\hline $1 \%$ & 750 & 0.9999 & 0.9999 & 0.9999 & 0.9989 & 0.9996 & 1.0000 & 0.9997 & 0.9997 \\
\hline $5 \%$ & 250 & 0.9974 & 0.9974 & 0.9971 & 0.9895 & 0.9938 & 0.9997 & 0.9963 & 0.9957 \\
\hline \multicolumn{10}{|c|}{ Size Simulation } \\
\hline$\underline{\mathrm{VaR}}$ & $\underline{\text { Sample }}$ & $\underline{\mathrm{LB}(1)}$ & $\underline{\mathrm{LB}(5)}$ & Markov & Weibull & Geometric & $\underline{\text { Caviar }}$ & $\underline{\mathrm{KS}}$ & $\underline{\text { CVM }}$ \\
\hline $1 \%$ & 250 & 0.9190 & 0.9190 & 0.9190 & 0.6896 & 0.7119 & 0.9189 & 0.9190 & 0.9190 \\
\hline $1 \%$ & 500 & 0.9937 & 0.9937 & 0.9937 & 0.9619 & 0.9664 & 0.9938 & 0.9937 & 0.9937 \\
\hline $1 \%$ & 750 & 0.9992 & 0.9992 & 0.9992 & 0.9949 & 0.9964 & 0.9994 & 0.9992 & 0.9992 \\
\hline $5 \%$ & 250 & 1.0000 & 1.0000 & 1.0000 & 1.0000 & 1.0000 & 1.0000 & 1.0000 & 1.0000 \\
\hline
\end{tabular}


Table 7: Backtesting Actual VaRs from Four Business Lines

\begin{tabular}{|c|c|c|c|c|c|c|c|c|}
\hline \multicolumn{9}{|c|}{ Business Line 1} \\
\hline & $\underline{\mathrm{LB}(1)}$ & $\underline{\mathrm{LB}(5)}$ & Markov & Weibul & $\underline{\text { Geometric }}$ & $\underline{\text { Caviar }}$ & $\underline{\mathrm{KS}}$ & $\underline{\text { CVM }}$ \\
\hline Get Volno & 0.0955 & 0.4830 & 0.1961 & 1.0138 & 1.2896 & 2.9296 & 18.7481 & 2.4382 \\
\hline -Value & 0.4601 & 0.5513 & 0.9628 & 0.6619 & 0.3758 & 0.3189 & 0.3241 & 0.3948 \\
\hline
\end{tabular}

Business Line 2

\begin{tabular}{|c|c|c|c|c|c|c|c|c|}
\hline & $\underline{\mathrm{LB}(1)}$ & $\underline{\mathrm{LB}(5)}$ & Markov & Weibull & Geometric & Caviar & $\underline{\mathrm{KS}}$ & CVM \\
\hline & & 0.1594 & & & 3.8377 & 389 & 11.5003 & \\
\hline & 0.8252 & 0.8379 & 0.3199 & 0.2354 & 0.1251 & 0.2339 & 0.4671 & 0.5618 \\
\hline
\end{tabular}

Business Line 3

\begin{tabular}{|c|c|c|c|c|c|c|c|c|}
\hline & $\underline{\mathrm{LB}(1)}$ & $\underline{\mathrm{LB}(5)}$ & Markov & Weibull & Geometric & $\underline{\text { Caviar }}$ & $\underline{\mathrm{KS}}$ & $\underline{\mathrm{CVM}}$ \\
\hline & & 0.0083 & 6.8487 & $\mathrm{NaN}$ & $\mathrm{NaN}$ & 27.7656 & 70.3651 & \\
\hline & 0.9920 & 0.9919 & 0.0176 & & & 0.0124 & 0.0533 & 0.024 \\
\hline
\end{tabular}

Business Line 4

\begin{tabular}{|c|c|c|c|c|c|c|c|c|}
\hline & $\underline{\mathrm{LB}(1)}$ & $\underline{\mathrm{LB}(5)}$ & Markov & Weibull & Geometric & Caviar & $\underline{\mathrm{KS}}$ & $\underline{\text { CVM }}$ \\
\hline & & 38.5720 & & & 4.9972 & 4.1304 & 19.6270 & \\
\hline & 78 & 0.0093 & 0.3687 & 0.1720 & 0.0602 & 0.2010 & 0.1815 & 0.1801 \\
\hline
\end{tabular}

